\title{
Fundamental Study on Operational Parameters of Diaphragmless Shock Tube
}

\author{
Masanori NISHIYAMA ${ }^{1}$, Masato TAGUCHI ${ }^{2}$ and Masashi KASHITANI ${ }^{3}$ \\ ${ }^{1,2,3}$ Department of Aerospace Engineering, National Defense Academy of Japan, 1-10-20 Hashirimizu Yokosuka, Kanagawa, 239-8686, \\ Japan
}

\begin{abstract}
This paper shows influences of initial conditions on a diaphragmless shock tube operation. This facility consists of a driver tube, a driven tube and a damp tank. The driver tube has a circular cross section with diameter of $150 \mathrm{~mm}$ and the driven tube, a rectangular cross section $(60 \mathrm{~mm} \times 150 \mathrm{~mm})$. The shock tube is operated by using a quick-opening pneumatic piston instead of a diaphragm. For the operation, pressure inside the pneumatic piston (piston pressure) is quickly released by opening a solenoid valve. In this paper, the initial piston pressure was chosen as a parameter to investigate effects on test flow conditions created by the shock tube. As a result, it was found that when the piston pressure at initial condition is large, piston pressure decreased more rapidly than that obtained for a small piston pressure condition, regardless of the pressure ratio of driver and the driven tube. In the condition of a constant initial operational pressure ratio and a different piston pressure, the shock Mach number was almost constant.
\end{abstract}

\section{Introduction}

It is well known that a shock tube is used for various researches including high-speed aerodynamics $[1,2]$. The shock tube of diaphragmless type is considered as the attractive aerodynamic devices because test flow is not contaminated by the minute fragments of the film. The quick-opening pneumatic piston was installed for diaphragmless operation of our shock tube. This shock tube system has been used to investigate aerodynamic characteristics of the transonic airfoil flows, and to develop the progress flow visualization technique for the past few years. For example, developing the focusing schlieren system to visualize slice image of transonic airfoil flows etc [3-5].

With the diaphragmless system, it is comparatively easy to adjust the Mach number and Reynolds number of test flow. Repeatability of the parameters is also better than conventional diaphragm-type shock tube, because the diaphragmless system remove uncertainties due to rupture of diaphragm. Test flow condition of shock tube is theoretically determined by differential pressure between high pressure driver section and low pressure driven section, and then arbitrary test flow conditions can be obtained by appropriate control of the initial pressure. The Shock Mach number $M_{\mathrm{s}}$ is determined by the pressure ratio between the high and low pressure section $p_{4} / p_{1}$. According to the theory, it is well understood that larger $p_{4} / p_{1}$ creates higher $M_{\mathrm{s}}$ [6]. However, in diaphragmless shock tube, $M_{\mathrm{s}}$ becomes smaller than theoretical value because the piston opens in a finite time.
Thus, opening behavior of the piston can induce an uncertainty of the test flow parameters.

Purpose of this study is to understand correlation between pressure inside of the pneumatic piston (piston pressure, $p_{\mathrm{p}}$ ) and shock Mach number $M_{\mathrm{s}}$. The diaphragmless shock tube is operated with several piston pressure to measure the shock Mach number and influences of the piston pressure on the shock Mach number is assessed. Furthermore, opening behavior of the piston is evaluated by monitoring history of piston pressure while the shock tube is operated.

\section{Experimental setup}

\subsection{Diaphramgless shock tube}

Dimension of the diaphragmless shock tube is shown in Fig.1. This facility consists of a driver tube, a driven tube and a damp tank. The driver tube (i.e. high pressure section, $p_{4}$ ) has a circular cross section with diameter of $150 \mathrm{~mm}$ and the driven tube (i.e. low pressure section, $p_{1}$ ), a rectangular cross section $(60 \mathrm{~mm} \times 150 \mathrm{~mm})$. The damp tank is installed at edge of the low pressure section. To measure wall pressure, pressure transducers can be flushmounted at eleven pressure taps which is created on wall of the low pressure section.

When the shock tube is operated, the initial pressure ratio $p_{4} / p_{1}$ of the high pressure chamber and the low pressure chamber is adjusted. And the partition part of the high pressure chamber and low pressure chamber is 


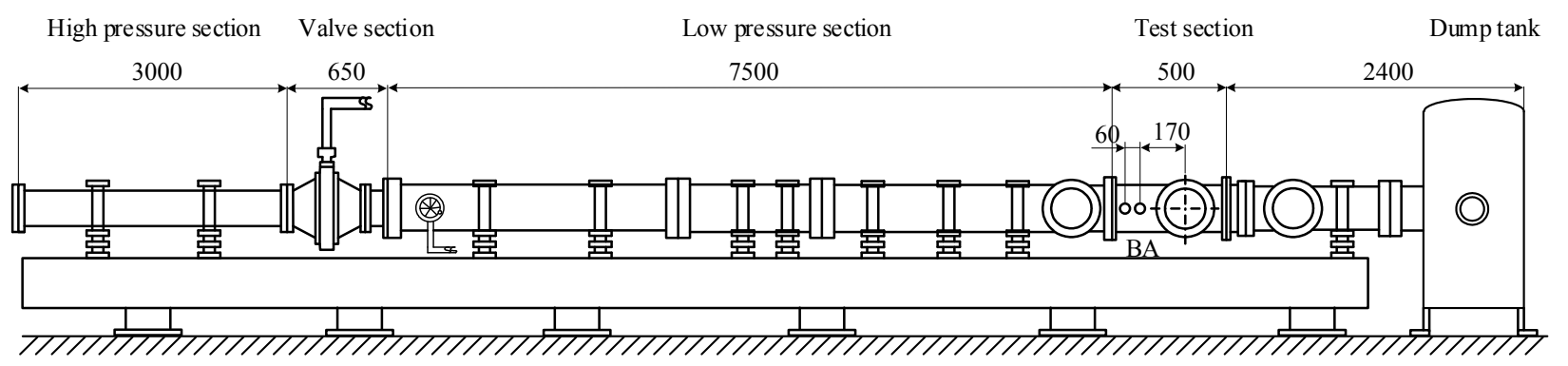

Figure 1. Diaphragmless Shock Tube[3].

quickly opened, immediately after, a primary shock wave is generated and propagates into the low pressure camber. To evaluate the test flow conditions, two PCB pressure transducers (H113A) are installed at tap A and B (see Fig.1). The PCB sensors can detect immediate pressure rise which is induced when the primary shock reaches the locus of the sensors. Shock Mach number $M_{S}$ is calculated from time difference between the pressure rise at the two pressure sensors by use of a universal counter (Pendulum, CNT-90).

A quick-opening pneumatic piston is installed to partition those two sections. The system of a quick opening valve was designed by Ref. [7]. The schematic diagram of the quick-opining valve of the shock tube is shown in Fig. 2. The valve consists of a piston, a solenoid valve that drives the piston quickly, and a leak section. When the shock tube is operated, pressure inside the piston is quickly released by opening a solenoid valve, and then the piston moves quickly due to pressure reduction in a short time.

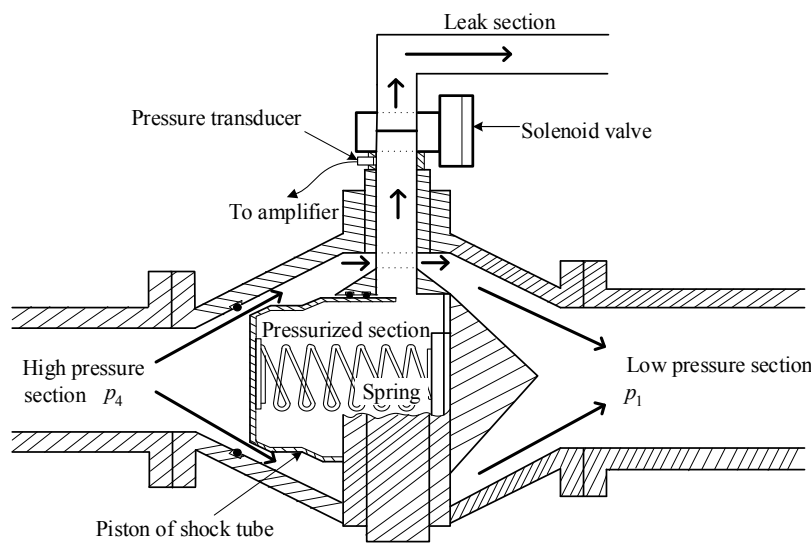

Figure 2. Quick-opening valve.

The pressure transducer is installed at the side wall just upstream of the solenoid valve. A Kulite pressure transducer (XT-190-250A) is used to monitor the pressure of the pneumatic piston $p_{\mathrm{p}}$. Time response of the piston movement is estimated from history of the pressure reduction due to the shock tube operation. The data of the pressure histories is measured by the digital oscilloscope (Tektronix, TDS 3000 series).

\subsection{Experimental condition}

Table 1 shows the experimental condition in this study. The conditions of three initial pressure ratios $p_{4} / p_{1}=60$, 100,150 are set across those two sections. The initial piston pressure $p_{\mathrm{p}}$ as the operational parameter is adjusted from 311 to $673 \mathrm{kPa}$. Case A shows the initial piston pressure on the measurement of the history $p_{\mathrm{p}}$, and Case $\mathrm{B}$ shows the condition for estimating the shock Mach number in the different initial pressure $p_{\mathrm{p}}$. Also, the shock Mach numbers measured in the study are shown in the table, and the result is discussed in section 3.2.

Table 1. Experimental condition.

\begin{tabular}{|c|c|c|}
\hline$p_{4} / p_{1}$ & Initial $p_{\mathrm{p}}$ & $M_{\mathrm{s}}$ \\
\hline 60 & $\begin{array}{l}\text { Case A: 475, 670, } 630 \\
\mathrm{kPa} \\
\text { Case B : } 311-663 \mathrm{kPa}\end{array}$ & $\begin{array}{c}1.63 \pm 0.04 \\
(1.59-1.66)\end{array}$ \\
\hline 100 & $\begin{array}{l}\text { Case A: 475, 670, } 630 \\
\mathrm{kPa} \\
\text { Case B : } 348-652 \mathrm{kPa}\end{array}$ & $\begin{array}{c}1.72 \pm 0.03 \\
(1.69-1.75)\end{array}$ \\
\hline 150 & $\begin{array}{l}\text { Case A: 475, 670,630 } \\
\text { kPa } \\
\text { Case A : } 350-650 \mathrm{kPa} \\
\text { Case B : } 345-673 \mathrm{kPa}\end{array}$ & $\begin{array}{c}1.78 \pm 0.04 \\
(1.74-1.82)\end{array}$ \\
\hline
\end{tabular}

\section{Results and discussion}

\subsection{Piston pressure history}

To understand opening behavior of the piston, the history of the piston pressure $p_{\mathrm{p}}$ is measured for initial piston pressures 475, 570 and $630 \mathrm{kPa}$. The shock tube operation pressure ratio $p_{4} / p_{1}$ is retained at 60,100 and 150 , respectively. The history of the piston pressure is plotted in Fig3 (a) to (c). The figures show the results of Case A. The horizontal axis represents the time from the initial condition, and the vertical axis represents the piston pressure $p_{\mathrm{p}}$. 


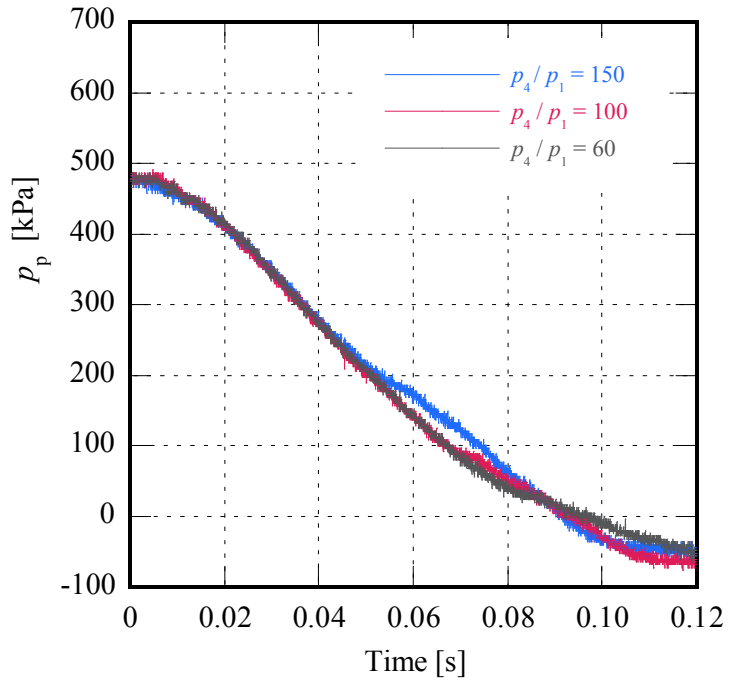

(a) $475 \mathrm{kPa}$

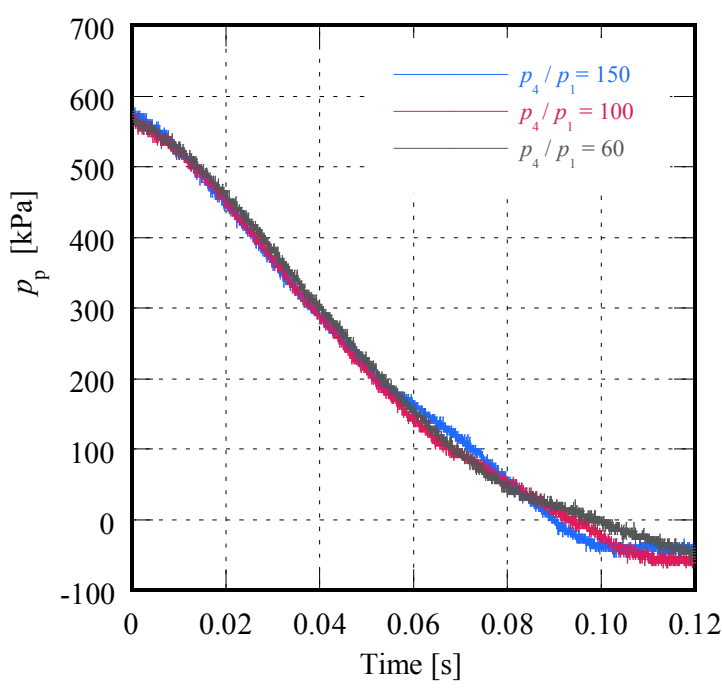

(b) $570 \mathrm{kPa}$

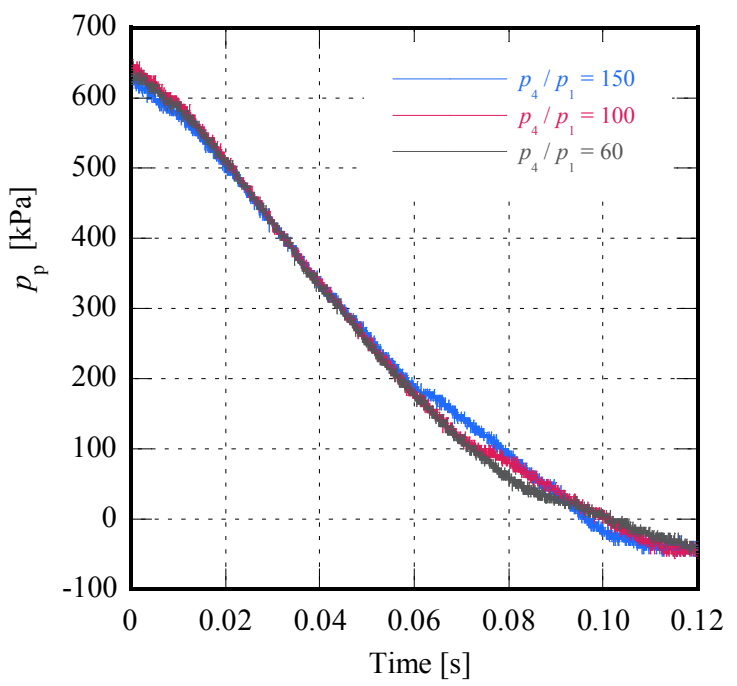

(c) $630 \mathrm{kPa}$

Figure 3. Piston pressure history.

(Effect of the operational pressure $p_{4} / p_{1}$ )

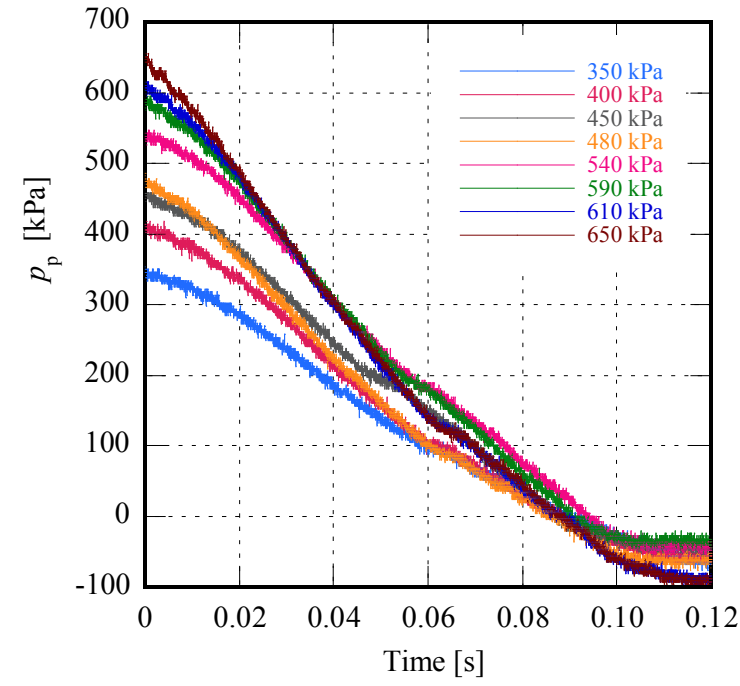

(a) Time history

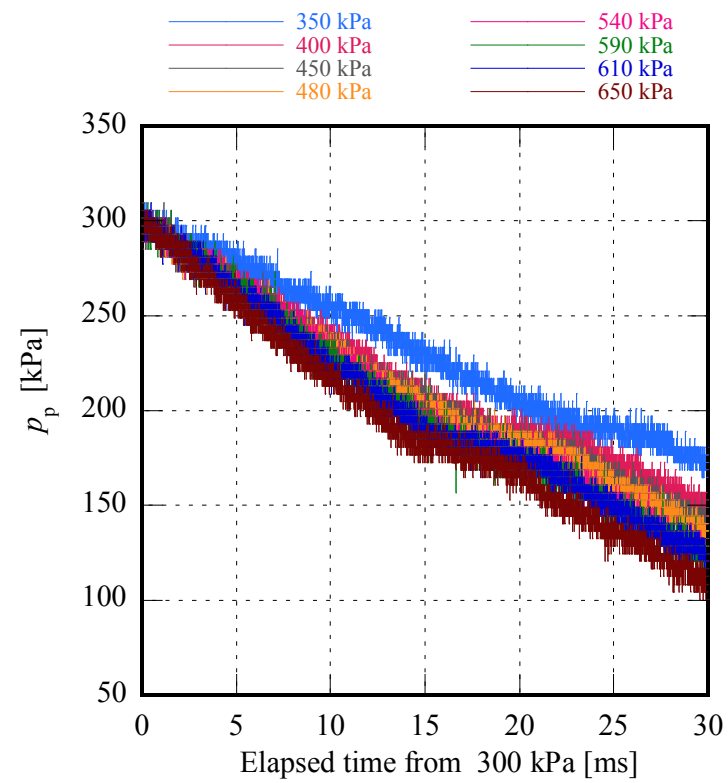

(b) Elapsed time history

Figure 4. Piston pressure history, $p_{4} / p_{1}=150$.

(Effect of the initial piston pressure $p_{\mathrm{p}}$ )

In Fig. 3 (a), it is confirmed that the piston pressure decreases from the initial pressure to 0 around $0.09 \mathrm{sec}$, and it is found that no significant difference is observed in those initial operational pressure ratios $p_{4} / p_{1}$. Also, it is still not clear under the differences between the three initial piston pressures (see Fig. 3 (b) and (c)). Therefore, the influence of the three initial operational pressure $p_{4} /$ $p_{1}$ did not greatly affect to the history of measured $p_{\mathrm{p}}$.

Fig. 4 (a) and (b) show the piston pressure history to understand the influence of the piston pressure $p_{\mathrm{p}}$. It is the experimental conditions of Case $\mathrm{B}$. The initial piston pressures are adjusted 350 to $650 \mathrm{kPa}$, the shock tube operational pressure ratio is set to $p_{4} / p_{1}=150$. Fig. 4 (a) shows the piston pressure history from 0 to $0.12 \mathrm{sec}$, and Fig. 4 (b) shows the elapsed time from $300 \mathrm{kPa}$. 


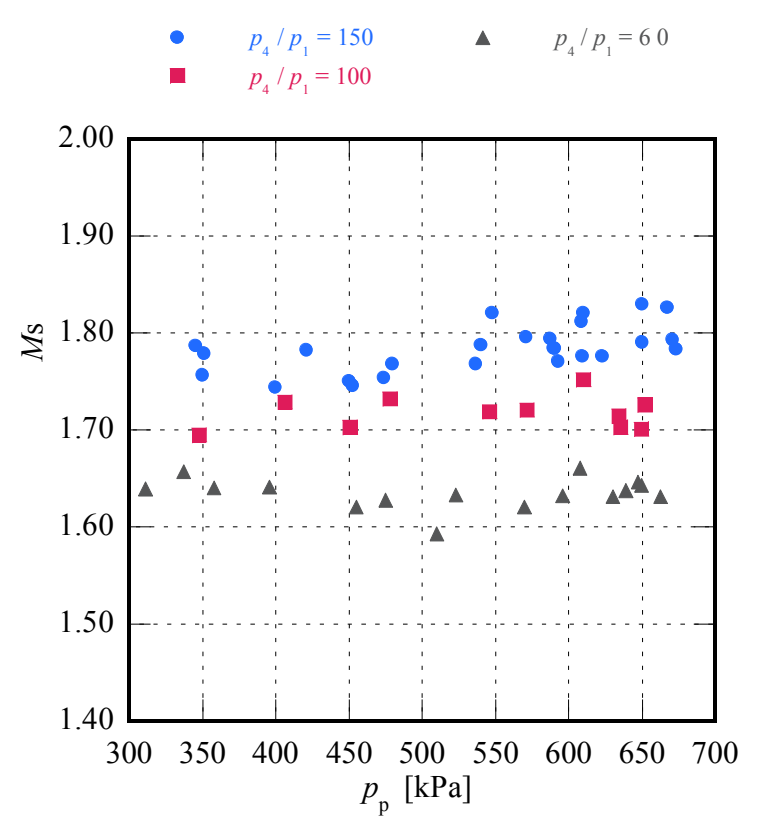

Figure 5. Shock Mach number.

For a large piston pressure at initial condition, piston pressure decreased more rapidly while piston moved than that obtained for a small piston pressure condition. And the all of the initial pressure conditions show 0 around $0.09 \mathrm{sec}$. It can be confirmed clearly in Fig. 4 (b).

\subsection{Shock mach number}

The shock Mach number $M \mathrm{~s}$ and pressure ratio $p_{4} / p_{1}$, which are measured under several piston pressure conditions $p_{\mathrm{p}}$, are plotted in Fig. 5. The horizontal axis represents the piston pressure $p_{\mathrm{p}}$ and the vertical axis represents the shock Mach number $M \mathrm{~s}$. Symbols indicate the data obtained at the shock tube operational pressure ratio $p_{4} / p_{1} 60,100$ and 150 respectively. The tendency shows that higher pressure ratio $p_{4} / p_{1}$ can create the higher shock Mach number. In $p_{4} / p_{1}=150$, even though the $M \mathrm{~s}$ is slightly scattering, but the shock Mach number is almost constant (the detail information is shown in Table 1). The same trend can be seen in $p_{4} / p_{1}=100$ and 60 , and this result indicates that the piston pressure do not have the impact on the determination of Ms. Also, the result shows the same trend as a previous study of a diaphragm-type shock tube [8].

\section{Conclusion}

This study was performed to understand the effect of an initial parameter for a diaphragmless shock tube which is driven by a pneumatic piston. Pressure inside the piston was employed as the test parameter. A number of tests were conducted under several piston pressure conditions, and shock Mach number and history of piston pressure were measured. The following results were obtained;

1) Pressure ratio $p_{4} / p_{1}$ does not greatly affect the history of piston pressure.

2) For a large piston pressure at initial condition, piston pressure decreased more rapidly while piston moved than that obtained for a small piston pressure condition.

3) The shock Mach number is almost constant, and the piston pressure do not have influence on the determination of $M \mathrm{~s}$.

\section{References}

1. William. J. Cook: Shock Tube as a Device for Testing Transonic Airfoils at High Reynolds Numbers, AIAA J., 17-7, (1979) pp.715-721.

2. H. Olivier, T. Reichel and M. Zechner: Airfoil Flow Visualization and Pressure Measurements in HighReynolds Number Transonic Flow", AIAA J., 41-8, (2003), pp. 1405-1412.

3. M. Kashitani, Y. Yamaguchi, G. Oki, H. Kitano and S. Esashi: Preliminary Study on Diaphragmless Shock Tube for Transonic Airfoil Testing with PDI, AIAA Paper 2010-744, 2010.

4. M. Kashitani, Y. Yamaguchi and H. Kitano, Preliminary Study for Quantitative Measurement of Flow Fields by Focusing Schlieren Method, AIAA Paper 2011-1983, 2011.

5. M. Kashitani, Y. Yamaguchi, D. Miyazaki and G. Oki: A Modified Point-Diffraction Interferometry for Shock Tube Airfoil Testing, AIAA Paper 2012-468, 2012.

6. T. Ikui, K. Matsuo: Mechanics of Shock Wave, Corona Publishing, (1983), pp.149-199 (In Japanese).

7. T. Otomo, T. Kuribayashi. and K. Takayama: The Characteristics of $100 \mathrm{~mm} \times 180 \mathrm{~mm}$ Diaphragmless Shock Tube after Improvement, 2002 Shock Wave Symposium, (2003) pp.7-8. (In Japanese).

8. K. Kuraya, S. Tsuchiya: Chemical physics of Shock Wave, Shyokabo Publishing, (1968), pp. 52-56 (In Japanese). 